\title{
La prueba de caminata en seis minutos en la evaluación de la capacidad de ejercicio en pacientes con enfermedad pulmonar obstructiva crónica
}

\author{
Carmen Lisboa B, Paulina Barría P, Jorge Yáñez V, \\ Marcia Aguirre Z, O rlando Díaz P. \\ Six minutes walk for the assessment \\ of patients with chronic obstructive \\ pulmonary disease
}

Exercise capacity can be evaluated in patients with chronic obstructive pulmonary disease (COPD), measuring the distance that patients are able to walk in 6 minutes (six-minute walk distance test; 6WDT). This test is simple to perform, inexpensive, reproducible and safe. It has been frequently employed for the assessment of COPD patients due to its high prognostic value of mortality and its usefulness to evaluate long-term of therapeutic interventions. In severe stages of the disease, the declining results of the best are useful to detect worsening. This review describes the method, standardization and reference values for the 6WDY and the results obtained with different therapeutic interventions, based on data from the literature and from the authors experience. We also review its predictive value for mortality and its value in the assessment of patients with more severe COPD (Rev Méd Chile 2008; 136: 1056-64).

(Key words: Diagnostic techniques, respiratory system; Pulmonary disease, chronic obstructive; Respiratory function tests)

Recibido el 8 de junio, 2007. Aceptado el 28 de septiembre, 2007.

Trabajo financiado por Proyectos FONDECYT 1951149, 1990513, 1010993 y 1030652.

Departamento de Enfermedades Respiratorias. Pontificia Universidad Católica de Chile. Santiago de Chile.

L a capacidad para realizar ejercicios se deteriora frecuentemente en los pacientes con enfermedad pulmonar obstructiva crónica (EPOC) debido a la disnea progresiva y al compromiso muscular periférico secundario al sedentarismo y a los efectos sistémicos de la enfermedad ${ }^{1}$. La disnea y la disminución de la capacidad de

Correspondencia a: Dra. Carmen Lisboa. Marcoleta 352, Primer Piso. Teléfono 633 1541. Fax 6335255. E mail: clisboa@med.puc.cl ejercicio condicionan, a su vez, una menor calidad de vida ${ }^{2}$. Por esto, las medidas terapéuticas aplicadas a los pacientes con EPOC deben tener entre sus objetivos centrales aliviar la disnea, aumentar la capacidad de ejercicio y mejorar la calidad de vida ${ }^{1}$.

La evaluación de la capacidad de ejercicio puede realizarse de las siguientes maneras: a) midiendo el consumo máximo de oxígeno $\left(\mathrm{VO}_{2}\right.$ max) o la carga máxima alcanzada durante un ejercicio progresivo limitado por síntomas, empleando una bicicleta ergométrica; b) determinan- 
do la tolerancia en el tiempo a un ejercicio en bicicleta ergométrica utilizando una carga submáxima constante; y c) midiendo la distancia que el paciente puede recorrer caminando en un tiempo predeterminado a su propia velocidad (caminata en 6 minutos) o bien caminando a velocidades progresivas que se aumentan cada 1 min en un corredor de 10 metros (shuttle-walk test). Los dos primeros métodos requieren de sistemas que midan la ventilación minuto, el consumo de oxígeno, la producción de $\mathrm{CO}_{2}$, el ciclo respiratorio, etc., además de personal capacitado, por lo que no están disponibles en todos los laboratorios de función pulmonar. Las pruebas que utilizan la caminata, y en particular la caminata en 6 minutos, han adquirido progresiva importancia por su fácil implementación y porque se asemejan más a las actividades de la vida diaria ${ }^{3}$.

Basados en los antecedentes de la literatura y en la experiencia de los autores, el presente artículo tiene como propósito describir las características de la prueba de caminata en 6 minutos (C6M), sus aplicaciones clínicas para evaluar la respuesta a intervenciones terapéuticas y su capacidad de predicción de gravedad y de deterioro de los pacientes con EPOC.

Antecedentes. En 1976, McGavin y cols ${ }^{4}$ describieron la prueba de caminata en 12 min para evaluar la capacidad de ejercicio en pacientes con enfermedades respiratorias. Posteriormente, Butland y cols $^{5}$ estudiaron la reproducibilidad y adaptabilidad de la prueba empleando dos, seis y doce minutos y concluyeron que la prueba de 6 minutos era la más reproducible y mejor tolerada. Esta prueba de caminata o distancia recorrida en 6 minutos (six minute walking distance [6MWD] test) de la literatura inglesa, ha adquirido gran importancia en la evaluación de la capacidad funcional en pacientes con enfermedades respiratorias y cardiovasculares, especialmente en aquellos portadores de EPOC ${ }^{3}$. La C6M es una prueba de ejercicio submáximo ${ }^{6}$, que se correlaciona estrechamente con el consumo máximo de $\mathrm{O}_{2}{ }^{7} \mathrm{y}$ posee las ventajas de no requerir equipos especiales, tener un bajo costo, buena reproducibilidad, ser de fácil aplicación y prácticamente carecer de riesgos $^{3}$. Además, por tratarse de caminar, actividad que todos realizamos en nuestra vida diaria, refleja mejor el estado funcional de los sujetos ${ }^{8}$. A ello se suma su capacidad predictiva de sobrevi$\mathrm{da}^{9}$, su capacidad para objetivar la respuesta a intervenciones terapéuticas y, su sensibilidad para detectar la progresión del deterioro de la EPOC avanzada $^{10}$. La Sociedad Americana de Tórax publicó en el año 2002 guías prácticas de estandarización de esta prueba basándose en los antecedentes disponibles hasta entonces ${ }^{3}$. Dicha comunicación incluye las indicaciones, contraindicaciones, limitaciones y bases racionales de la prueba, así como las medidas de seguridad y los aspectos técnicos que deben considerarse. Una de las consecuencias de su publicación, cuya lectura recomendamos, es que promovió numerosos estudios destinados a aclarar las áreas de incertidumbre de la C6M. Dichos estudios terminaron por confirmar su utilidad clínica.

Respuesta fisiológica a la prueba. El perfil de consumo de oxígeno $\left(\mathrm{VO}_{2}\right)$ de la C6M corresponde al de un ejercicio submáximo, caracterizado por una meseta del $\mathrm{VO}_{2}$ sin alcanzar el $\mathrm{VO}_{2}$ $\max ^{6,11}$. En pacientes con EPOC se ha observado además que esta meseta incluye a la ventilación minuto y la frecuencia cardiaca, ocurre aproximadamente a partir del tercer minuto de la prueba ${ }^{12}$, y correspondería a la carga $0 \mathrm{VO}_{2}$ crítico que puede sostener el paciente por un período largo. Esto fue planteado debido a que los pacientes estudiados adoptaban una velocidad alta pero constante durante la prueba ${ }^{12}$. La velocidad constante de marcha fue también observada en nuestro laboratorio por Pinochet y cols ${ }^{13}$ en pacientes con EPOC, demostrando que si utilizaban la misma velocidad que emplearon durante la caminata en una cinta rodante, los índices medidos al final de ambas pruebas eran similares.

Método y estandarización. La C6M consiste en caminar lo más rápidamente posible en un corredor plano, idealmente de $30 \mathrm{~m}$ de longitud, durante 6 minutos. En este período los pacientes son alentados cada minuto por el operador en una forma previamente establecida ("lo está haciendo muy bien; siga así, por ejemplo) y se les informa también del tiempo transcurrido y del que resta para completar la prueba. La diferencia entre la distancia caminada con y sin estímulo verbal supera en promedio los 30 metros $^{14}$ por lo que se debe alentar a los pacientes durante su desarrollo. 
$\mathrm{Si}$ el paciente se detiene antes de transcurrir los 6 minutos, ya sea por disnea, fatiga de extremidades inferiores u otros motivos, el tiempo sigue contabilizándose durante la detención y el paciente puede o no reanudar la marcha para completar los seis minutos. La prueba debe repetirse luego de un período de descanso de al menos media hora, pues se ha demostrado que existe algún grado de aprendizaje. Esto determina que la distancia recorrida en la segunda prueba sea generalmente mayor que en la primera. En cambio, una tercera prueba no aumenta la distancia recorrida.

Al inicio y final de la $\mathrm{C} 6 \mathrm{M}$ se evalúa la magnitud de la disnea, empleando la escala psicofísica de Borg ${ }^{15}$, que posee un rango de $0 \mathrm{a}$ 10 puntos, siendo cero la ausencia total de dificultad respiratoria y 10, el máximo posible. La escala de Borg se emplea también para evaluar la magnitud de la fatiga de las extremidades inferiores. Habitualmente se mide la saturación de $\mathrm{O}_{2}$ $\left(\mathrm{SaO}_{2}\right)$ y la frecuencia de pulso al inicio y final de la prueba. La distancia recorrida se obtiene contabilizando el número de vueltas recorridas en forma completa y los metros finales donde el sujeto se detuvo al cumplirse los 6 minutos.

Valores normales. Mientras que el método y la estandarización de la prueba han sido ampliamente detallados ${ }^{3}$, los valores de referencia existentes son escasos y relativamente recientes ${ }^{16-19}$. El primero de estos estudios reclutó 290 sujetos entre 40 y 80 años, pero sólo se realizó una prueba y los sujetos caminaron a su propio paso, sin estímulo verbal $^{16}$. Con estas ecuaciones, los valores de referencia son inferiores a los propuestos en dos estudios posteriores ${ }^{17,18}$, probablemente porque en estos últimos los sujetos fueron alentados verbalmente, y la prueba se repitió $\operatorname{dos}^{17} \mathrm{y}$ cuatro ${ }^{18}$ veces, considerándose la mayor distancia observada para generar las ecuaciones. La distancia recorrida fue mayor en la segunda prueba en ambos estudios, en $8 \pm 5 \% 17$ y 43 metros $^{18}$. Desafortunadamente el número de pacientes reclutados fue relativamente pequeño: 53 sujetos entre 53 y 85 años ${ }^{17}$ y 79 sujetos entre 20 y 80 años ${ }^{18}$, respectivamente. Por último, Casanova y cols $^{19}$ recientemente finalizaron un estudio multicéntrico realizado España y Sudamérica en sujetos normales entre 40 y 80 años, con el fin de obtener valores de referencia en una población más numerosa. El análisis preliminar de estos datos ha demostrado que la C6M depende de la edad y del género, y que asimismo es mayor en los sudamericanos que en los españoles.

En la Tabla 1 se describen los valores teóricos de C6M para un hombre y una mujer con características antropométricas típicas de nuestra población de acuerdo a las ecuaciones de referencia más frecuentemente utilizadas ${ }^{16,17}$. Se incluye la ecuación obtenida en conjunto en dos centros de nuestro país (observaciones no publicadas).

Efecto de las intervenciones terapéuticas. El impacto de la EPOC en un paciente individual sólo depende parcialmente del grado de compromiso del $\mathrm{VEF}_{1}$, existiendo otros factores importantes que también condicionan la magnitud de la disnea y de la limitación para realizar ejercicios. Estos hechos

\section{Tabla 1. Caminata en 6 minutos predicha por tres ecuaciones de referencia para un hombre y una mujer de características antropométricas promedio en Chile}

\begin{tabular}{|lcc|}
\hline Ecuación (Primer autor) & \multicolumn{2}{c|}{$\begin{array}{c}\text { Caminata en } 6 \text { minutos (metros) } \\
\text { Mujer }^{\ddagger}\end{array}$} \\
\hline Enright & 554 & 521 \\
Troosters & 698 & 600 \\
Barría* & 619 & 574 \\
\hline
\end{tabular}

${ }^{\dagger}$ Hombre de 60 años, estatura $170 \mathrm{~cm}$ y peso $70 \mathrm{~kg}$.

₹ Mujer de 60 años, estatura $156 \mathrm{~cm}$ y peso $56 \mathrm{~kg}$.

* Se emplearon los datos de 40 hombres y 40 mujeres entre 40 y 80 años obtenidos en Chile (observaciones no publicadas de Barría P, Yáñez J, Palacios S, Díaz O, Lisboa C). 
apoyan la política actual de centrar el tratamiento de la enfermedad en el alivio de los síntomas, los cuales también deben ser utilizados en la evaluación de la terapia y de la progresión de la EPOC. La C6M constituye un elemento simple y valioso para la evaluación clínica de la enfermedad.

- Cambio mínimo clínicamente importante

El cambio mínimo clínicamente importante (MCI) de la caminata en 6 minutos fue establecido por Redelmeir y cols ${ }^{20}$, quienes consignaron una media de $54 \mathrm{~m}$ con un intervalo de confianza de 95\% (IC 95\%) entre 30 y $72 \mathrm{~m}$. Estos valores se han utilizado para establecer si una intervención produce cambios clínicos que puedan ser considerados como beneficiosos para el paciente.

\section{- Broncodilatadores}

La administración aguda de broncodilatadores produce resultados variables sobre la $\mathrm{C}_{6} \mathrm{M}^{21}$. El efecto de bromuro de ipratropio fue evaluado en nuestro grupo por Jorquera y $\operatorname{cols}^{22}$, quienes observaron un aumento clínicamente significativo de la C6M junto a una recuperación más rápida de la disnea y de la hiperinflación pulmonar. En un estudio posterior, observamos que al agregar durante dos semanas teofilina a broncodilatadores inhalados de acción corta se producía un aumento clínicamente relevante de la C6M en $42 \%$ de los pacientes tratados y sólo en $5 \%$ de los controles, junto con una mejoría significativa de la hiperinflación pulmonar y de la disnea ${ }^{23}$.
Los estudios multicéntricos destinados a investigar la eficacia de los broncodilatadores $\aleph_{2}$ adrenérgicos de larga duración, asociados 0 no a corticoides inhalados, no han evaluado el efecto de la administración de estos fármacos sobre la C6M. Por otro lado, aunque el efecto de tiotropio sobre la capacidad de ejercicio ha sido estudiado por varios autores, sólo uno de ellos incluyó la caminata en 6 minutos en la evaluación funcional, la que aumentó significativamente comparada con place$\mathrm{bo}^{24}$.

Por los antecedentes mencionados, no parece recomendable emplear la C6M para evaluar el efecto agudo de los broncodilatadores. Sin embargo, consideramos que en estudios multicéntricos destinados a determinar la eficacia de drogas administradas por largos períodos, la inclusión de resultados centrados en el paciente, como es la C6M debería ser considerada.

\section{- Rehabilitación pulmonar}

El entrenamiento físico es el componente fundamental de la rehabilitación pulmonar, siendo capaz de reducir la disnea, aumentar la capacidad de ejercicio y mejorar la calidad de vida de los pacientes sin modificar la función pulmonar. Aunque los efectos de la rehabilitación sobre la capacidad de ejercicio se ha evaluado generalmente con un cicloergómetro, varios ensayos clínicos han demostrado un aumento significativo de la $\mathrm{C}^{2} \mathrm{M}^{25,26}$. La Figura 1 muestra los efectos de estos programas, sugiriendo que aquellos con más

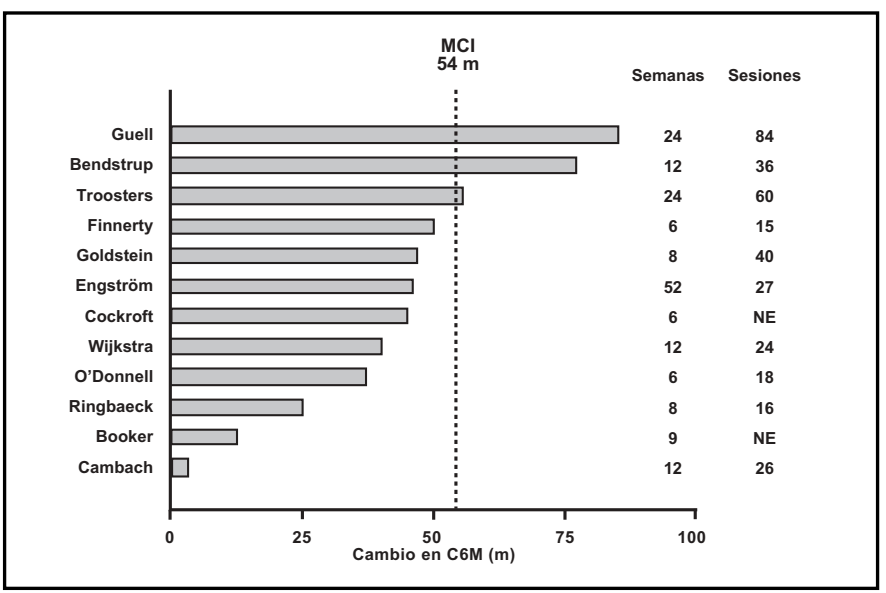

Figura 1. Resumen de las diferencias en la caminata en 6 minutos (C6M) entre los grupos tratados con rehabilitación y sus controles. Se incluyen los 12 estudios publicados donde este efecto es descrito. La línea punteada vertical representa el cambio mínimo clínicamente importante (MCI). Modificado de referencia 25. 
sesiones (>28) tienen más efectos sobre la C6M (50 vs $35 \mathrm{~m}$ ). Un meta análisis que incluye estos estudios sugiere además que los programas con duración $\geq 24$ semanas aumentan más la C6M que los programas de menor duración ${ }^{25}$. Lo mismo sucede cuando los programas incluyen supervisión directa de los pacientes.

- Entrenamiento muscular inspiratorio

El efecto del entrenamiento muscular inspiratorio (EMI) sobre la C6M ha sido evaluado en varios protocolos ${ }^{26-29}$. Nuestra experiencia demuestra que este procedimiento terapéutico es capaz de aumentar la C6M por sobre el mínimo clínicamente importante en los pacientes con EPOC $^{29}$. La Figura 2 muestra los cambios de la C6M obtenidos con el EMI que superaron el MCI en 4 de los 7 trabajos en que fue medida ${ }^{29}$. Todos estos estudios se caracterizaron por haber sido controlados por placebo, haber empleado una válvula umbral para el entrenamiento, y haber usado una carga inspiratoria de entrenamiento $\geq 30 \%$ a la presión inspiratoria máxima. La respuesta desigual de la C6M al EMI se explicaría en parte por la heterogeneidad de los pacientes a quienes se ha aplicado ${ }^{26}$. Esto ha sido sugerido por un meta análisis reciente, donde se observó que los pacientes con presiones inspiratorias máximas $\leq 60$ $\mathrm{cm} \mathrm{H}_{2} \mathrm{O}$ eran los que obtenían mayores benefi$\operatorname{cios}^{30}$.

- Administración de oxígeno

Los pacientes con EPOC avanzada frecuentemente presentan hipoxemia durante el ejercicio simultáneamente con disnea desproporcionada que limita su capacidad física. En algunos de ellos la administración de oxígeno disminuye la magnitud de la disnea, evita o aminora la caída de la $\mathrm{SaO}_{2}$ y aumenta la capacidad de ejercicio. De hecho, la hipoxemia marcada durante el ejercicio constituye una indicación de oxigenoterapia. La C6M ha demostrado ser más sensible a los cambios de la $\mathrm{SaO}_{2}$ que el ejercicio en bicicleta, probablemente porque el número de grupos musculares involucrados en el acto de caminar es mayor $^{31}$. Esto ha determinado que la C6M sea el tipo de ejercicio recomendado para prescribir el uso de oxígeno durante la deambulación ${ }^{2}$.

Sin embargo, el efecto del oxígeno sobre la capacidad de ejercicio es variable. Díaz y $\operatorname{cols}^{32}$ estudiaron 53 pacientes con EPOC a los cuales se les administró aleatoriamente $\mathrm{O}_{2} \mathrm{O}$ aire comprimido en

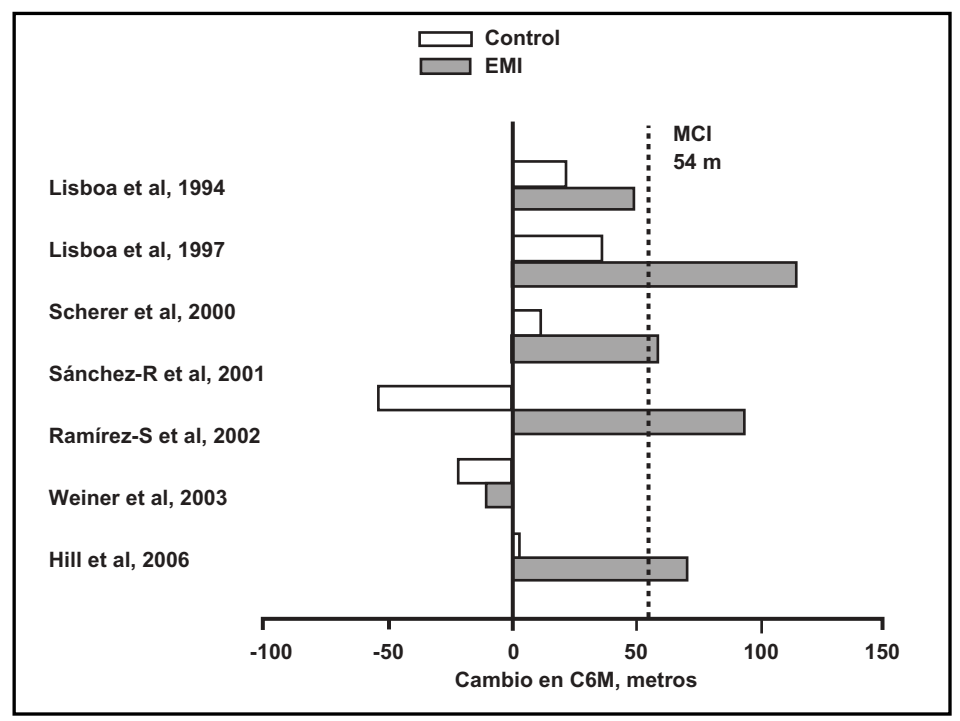

Figura 2. Efectos del entrenamiento muscular inspiratorio (EM) sobre la caminata en 6 minutos (C6M). Se comparan los cambios en la C6M entre controles (barras blancas) y tratados (barras grises) en 7 ensayos clínicos randomizados. La línea punteada vertical representa el cambio mínimo clínicamente importante (MCI). Modificado de referencia 29. 
reposo y luego durante la prueba de C6M. Observaron que los pacientes que tenían limitación del flujo espiratorio e hiperinflación pulmonar en reposo mientras respiraban aire disminuyeron la hiperinflación y aumentaron la C6M por sobre el MCI. Estos resultados explicarían al menos en parte la respuesta variable a la administración de oxígeno observada en la $\mathrm{EPOC}^{33}$.

- Ventilación no invasiva en EPOC estable

La ventilación no invasiva (VNI) aplicada a pacientes estables e hipercápnicos puede disminuir la $\mathrm{PaCO}_{2}$, la hiperinflación pulmonar y la disnea ${ }^{34}$. Su efecto sobre la C6M ha sido comunicado sólo en algunos estudios clínicos controlados, con resultados variables, por lo que un meta análisis reciente concluye que el beneficio sobre la capacidad de ejercicio está probablemente restringido a un grupo seleccionado de pacientes ${ }^{35}$. Nuestros datos sugieren que, bajo supervisión en el hospital a pacientes ambulatorios, la mejoría de la C6M atribuible a la VNI puede superar el MCI y ser evidente después de sólo tres semanas de empleo ${ }^{36}$.

Cirugía de reducción de volumen y transplante pulmonar. En esta área la C6M tiene una importante aplicación porque constituye uno de los criterios de inclusión para el trasplante pulmonar en EPOC y para la cirugía de reducción de volumen pulmonar ${ }^{37,38}$. En ambos casos se exige que la C6M preoperatoria, obtenida después de rehabilitación pulmonar, sea superior a $200 \mathrm{~m}$, dado que la mortalidad aumenta exageradamente bajo este punto de corte. La C6M también se puede emplear para evaluar los efectos de la cirugía de reducción de volumen, aunque no se ha establecido si es superior a las pruebas de ejercicio en cicloergómetro.

Predicción de la sobrevida. Pinto-Plata y asocia$\operatorname{dos}^{9}$ demostraron recientemente en una cohorte de 198 pacientes con EPOC grave seguidos por dos años, que la C6M predice mejor la mortalidad que otras dos variables con capacidad predictiva reconocida, como son el $\mathrm{VEF}_{1}$ y el estado nutricional. Los pacientes que sobrevivieron tenían al inicio del estudio una C6M significativamente mayor y una menor reducción anual de la C6M que los pacientes fallecidos. Los autores emplearon categorías de $100 \mathrm{~m}$ para clasificar la capacidad de ejercicio basal y demostraron que cada categoría tenía diferencias significativas en la sobrevida. Así, en aquellos pacientes que al inicio del estudio caminaban menos de 100 metros la mortalidad fue $\sim 80 \%$, mientras que los que caminaban $>300 \mathrm{~m}$ registraron una mortalidad menor al 20\% (Figura 3). Estos datos concuerdan

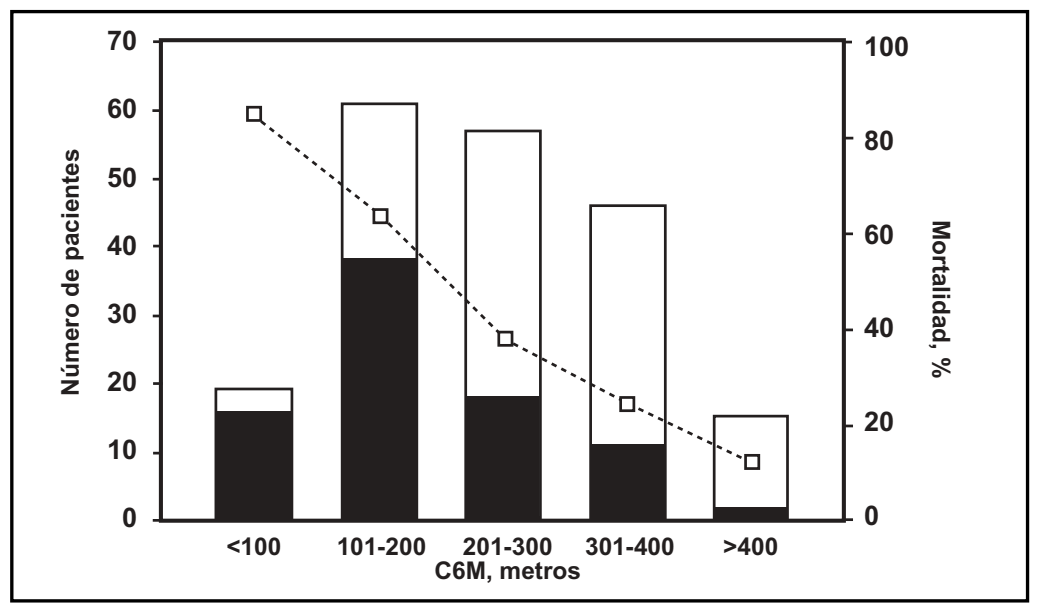

Figura 3. Caminata en 6 minutos y mortalidad. Gráfico de barras superpuestas que muestra a los pacientes divididos en cinco categorías de acuerdo a la distancia recorrida en una caminata de 6 minutos (C6M). El alto de la columna representa la número total de pacientes en cada categoría, mientras que la porción negra al número de fallecidos. Los símbolos unidos por la línea discontinua representan los porcentajes de mortalidad para cada categoría. Modificado de referencia 9. 
con los resultados de Martínez y cols ${ }^{39}$ que demostraron que caminar menos de $300 \mathrm{~m}$ aumentaba al doble el riesgo de muerte en la EPOC.

Evaluación del deterioro funcional. Tradicionalmente el deterioro progresivo del paciente con EPOC se ha medido a través de la caída del $\mathrm{VEF}_{1}$. Pinto-Plata y asociados ${ }^{9}$ estudiaron la declinación de la C6M durante 2 años en un grupo de pacientes con EPOC y observaron una caída de 40 $\mathrm{m} / \mathrm{año}$ en los que fallecieron vs. $22 \mathrm{~m} / \mathrm{año}$ en los sobrevivientes. Casanova y cols ${ }^{10}$ también observaron una reducción anual de la C6M en un estudio de seguimiento de 3 a 5 años en 294 pacientes. Sin embargo, esta fue significativa sólo en los pacientes con enfermedad más avanzada, es decir, con $\mathrm{VEF}_{1}<50 \%{ }^{2}$. La declinación fue de $19 \%$ en los graves, de $26 \%$ en los muy graves y de sólo $2 \%$ en los pacientes con enfermedad moderada. En cambio, el descenso del $\mathrm{VEF}_{1}$ fue mayor en los pacientes con enfermedad moderada y escaso en los muy graves. Una observación semejante hemos apreciado en una cohorte de pacientes ex fumadores con EPOC de moderada a muy grave que hemos seguido por 3 años ${ }^{40}$, como se observa en la Figura 4. En esta misma cohorte, al definir el deterioro clínico basados en un aumento de 4 puntos del puntaje global del cuestionario de calidad de vida de St George's, encontramos que los pacientes que se deterioraban exhibía una reducción del $\mathrm{VEF}_{1}$ de sólo $5 \%$ en relación al basal, mientras que la $\mathrm{C} 6 \mathrm{M}$ fue mucho más sensible, reduciéndose un $23 \%$. Los resultados del estudio de Casanova y cols ${ }^{10}$, al igual que los nuestros, destacan la importancia de emplear otros índices funcionales además del $\mathrm{VEF}_{1}$ para evaluar el deterioro de los pacientes con EPOC avanzada.

En suma, la C6M proporciona una información más valiosa y más relacionada con la calidad de vida que el $\mathrm{VEF}_{1}$ para establecer la gravedad de estos pacientes.

Índice multidimensional de gravedad BODE. Celli y cols $^{41}$ desarrollaron recientemente un índice multidimensional de gravedad para pacientes con EPOC, denominado BODE (Body mass index; airflow Obstruction; Dyspnea; Exercise performance). En su elaboración se incluye el índice de masa corporal, el $\mathrm{VEF}_{1}$, la disnea según la escala modificada del MRC (Medical Research Council) y la C6M. El BODE ha demostrado una capacidad predictiva de sobrevida mayor que cada uno de sus cuatro componentes por separado. Asimismo, en los estudios en que se ha empleado para evaluar el efecto de acciones terapéuticas, como

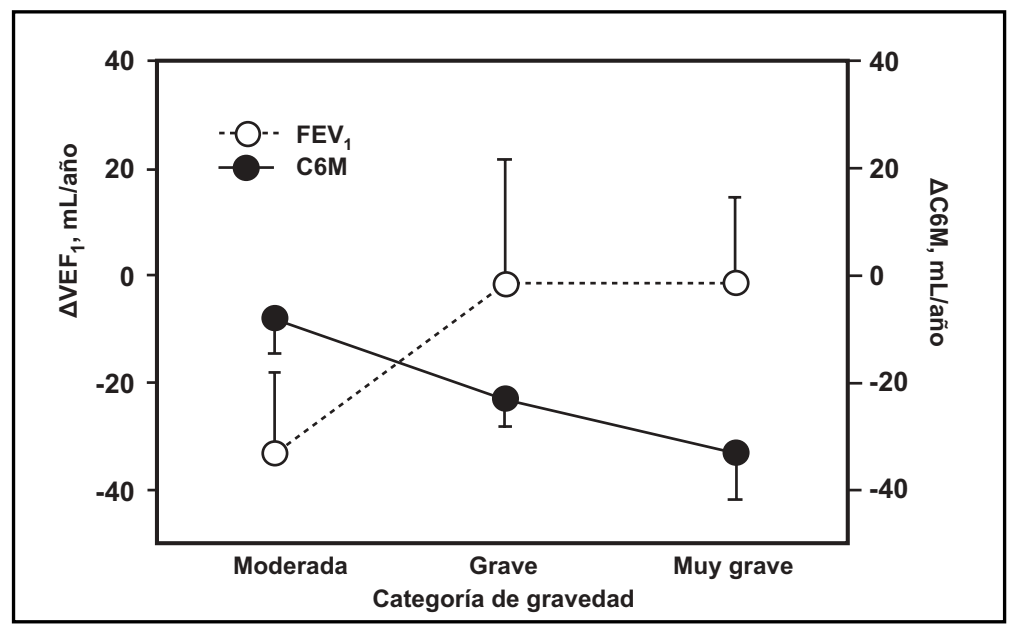

Figura 4. En un grupo de 66 pacientes con enfermedad pulmonar obstructiva crónica seguidos por 3 años, el $\mathrm{VEF}_{1}$ se deterioró más marcadamente en los pacientes con enfermedad moderada de acuerdo a la clasificación de las Sociedades Americana y Europea de Tórax $\left(\mathrm{VEF}_{1} 50-80 \%\right.$ del teórico). Por el contrario, la caminata en 6 minutos (C6M) apenas se redujo en este mismo grupo, pero se deterioró notoriamente en los pacientes con enfermedad grave y muy grave. Datos obtenidos de referencia 40. 
la rehabilitación pulmonar ${ }^{42}$, en la que el aumento de la C6M y el alivio de la disnea constituyen los logros más importantes, la mejoría del BODE se ha asociado a una importante reducción en la mortalidad de estos pacientes. En pacientes sometidos a cirugía de reducción de volumen pulmonar, Infeld y cols ${ }^{43}$ demostraron que el índice BODE medido después de la cirugía es un excelente predictor de sobrevida en sus pacientes.

Utilidad clínica y conclusiones. Del análisis de esta revisión se puede concluir que son varias las razones para incorporar a la clínica esta prueba de ejercicio simple: 1) Permite evaluar la gravedad de

\section{REFERENCIAS}

1. Rabe KF, Hurd S, Anzueto A, Barnes PJ, Buist SA, CALVERLEY P ET al. Global strategy for the diagnosis, management, and prevention of COPD - 2006 Upadate. Am J Respir Crit Care Med. Published Online First: 16 May 2007. doi: 10.1164rccm.200703-456SO.

2. Calverley PMA. Exercise and dyspnea in COPD. Eur Respir Rev 2006; 15: 72-9.

3. American Thoracic Society Statement. Guidelines for the six-minute walk test. Am J Respir Crit Care Med 2002; 166: 111-7.

4. McGavin CR, GuPTA SP, Mchardy GRJ. Twelve minute walking test for assessing disability in chronic bronchitis. BMJ 1976; 1: 822-3.

5. Butland RJA, Pamg J, Gross ER, Woodcock AA, GeDDES DM. Two, six and twelve-minute walking tests in respiratory diseases. BMJ 1982; 284: 1607-8.

6. Rabinovich RA, Vilano J, Roca J. Evaluación de la tolerancia al ejercicio en pacientes con EPOC. Prueba de marcha en 6 minutos. Arch Bronconeumol 2004; 40: 80-5.

7. Cahaln L, Pappagianopoulos P, Prevost S, Wain J, GinNs L The relationship of the 6- min walk test to maximal oxygen consumption in transplant candidates with end- stage lung disease. Chest 1995; 108: 452-9.

8. Carter R, Holday dB, Nwasuruba C, Stocks J, GroTHUES C, TIEP B. 6-minute walk work for assessment of functional capacity in patients with COPD. Chest 2003; 123: 1408-15.

9. Pinto-Plata VM, Cote C, Cabral H, taylor J, Cem BR. The 6 -min walk distance; change over time and value as a predictor of survival in severe COPD. Eur Respir J 2004; 23: 28-33.

10. Casanova C, Cote CG, Marin JM, De torres JP, Aguirre-Jaime A, Mendez R, Dordeuy L, Cew BR. The la enfermedad centrándose en la limitación de la capacidad de ejercicio y objetivando su deterioro en el tiempo, por lo que su medición a lo largo de la evolución de la EPOC complementa la información de la espirometría; 2) Sus cambios con las intervenciones terapéuticas proporcionan información centrada en el paciente sobre el beneficio clínico logrado; 3) El aumento de la C6M con la administración de $\mathrm{O}_{2}$ constituiría una forma objetiva para indicar oxigenoterapia en pacientes que sólo tienen hipoxemia y disnea en ejercicio; y 4) Permite recomendar fundadamente la rehabilitación pulmonar o la mantención de actividad física domiciliaria.

six- minute walk distance: Long term Follow up in patients with COPD. Eur Respir J 2007; 29: 535-40.

11. Casas A, Vilaro J, Ravinovich R, Mayer Am, Barberá JA, Rodriguez-Roisin R, Roca J. Encouraged 6-min walking test indicates maximum sustainable exercise in COPD patients. Chest 2005; 128: 55-61.

12. Troosters T, Vilaro J, Rabinovich R, Casas A, Barberá JA, Rodríguez-Roisin R ET AL. Physiological responses to the 6-min walk test in patients with chronic obstructive pulmonary disease. Eur Resp J 2002; 20: 564-9.

13. Pinochet R, Díaz O, Leiva A, Borzone G, Lisboa C. Adaptación de la prueba de caminata en 6 minutos en corredor a cinta rodante en pacientes con enfermedad pulmonar obstructiva crónica. Kinesiología, Revista Oficial del Colegio de Kinesiólogos de Chile 2003; 72: 69-72.

14. Guyatt GH, Pugsley SO, Suluvan MJ, Thompson PJ, Berman LB, Jones NL, FaLen EL, TaYlor DW. Effect of encouragement on walking test performance. Thorax 1984; 39: 818-22.

15. Borg GAV. Psychophysical basis of perceived exertion. Med Sci Sports Exerc 1982; 14: 377-81.

16. EnRight PL, Sherrill DL. Reference equations for the six-minute walk in healthy subjects. Am J Respir Crit Care Med 1998; 158: 1384-7.

17. Troosters T, Gosselink S, Decramer M. Six minute walking distance in healthy elderly subjects. Eur Respir J 1999; 14: 270-4.

18. Gibbons WJ, Fruchter N, SLoan S, Levy RD. Reference values for a multiple repetition 6-minute walk test in healthy subjects older than 20 years. J Cardiopulm Rehabil 2001; 21: 87-93.

19. Casanova C, López MV, Marin JM, De Torres JP, Casas A, Montes De Oca M. Six minute walk distance in a multicenter study of healthy subjects aged 40-80 years in Spain and South America. Am J Respir Crit Care Med 2007; 175: A955. 
20. Redelmeier DA, Bayoumi AM, Goldstein RS, Guyatt $\mathrm{GH}$. Interpreting small differences in functional status: the six minute walk test in chronic lung disease patients. Am J Respir Crit Care Med 1997; 155: $1278-82$.

21. Liesker JLW, Wijkstra PJ, Ten Hacken NHT, Köetergh, Postma DS, Kerstjens HAM. Systematic review of the effects of bronchodilators on exercise capacity in patients with COPD. Chest 2002; 121: 597-608.

22. Jorquera J, Díaz O, Lisboa C. Efecto del bromuro de ipratropio sobre la hiperinflación dinámica en pacientes con EPOC. Rev Méd Chile 2003; 131: 605-12.

23. Dreyse J, Silva F, Díaz O, Borzone G, Lisboa C. Beneficios clínicos y funcionales de agregar teofilina a la terapia inhalatoria con broncodilatadores de acción corta en pacientes con enfermedad pulmonar obstructiva crónica. Rev Méd Chile 2005; 133: 1211-9.

24. Verkindre C, Bart F, Aguilaniu B, Fortin F, Guerin J-C, Le MERRE C ET AL. The effect of tiotropium on hypeninflation and exercise capacity in chronic obstructive pulmonary disease. Respiration 2006; 73: 420-7.

25. Troosters T, Casaburi R, Gosselink R, Decramer M. Pulmonary rehabilitation in chronic obstructive pulmonary disease. Am J Respir Crit Care Med 2005; 172: 19-38.

26. Ries AL, Bauldoff GS, Carlin BW, Casaburi R, Emery CF, MAHLER DA ET AL. Pulmonary rehabilitation: Joint ACCP/AACVPR Evidence- based clinical practice guidelines. Chest 2007; 131: 4s-43s.

27. Lisboa C, Borzone G, Cruz E. Entrenamiento muscular inspiratorio en pacientes con enfermedad pulmonar obstructiva crónica. Rev Méd Chil 1998; 126: 563-8.

28. Lisboa C, Viliafranca C, Leiva A, Cruz E, Pertuze J, Borzone G. Inspiratory muscle training in chronic airflow limitation: effect on exercise performance. Eur Respir J 1997; 10: 537-42.

29. Lisboa C, Borzone G. Entrenamiento muscular inspiratorio en EPOC. Pulmón 2005; 5: 95-102.

30. Lötters F, Van Tol B, Kwakkel G, Gosseunk R. Effects of controlled inspiratory muscle training in patients with COPD: a meta-analysis. Eur Respir J 2002; 20: 570-7.

31. Poulain M, Durand F, Palomba B, Ceugniet F, Desplan J, VarRaY A, PréFaut C. 6-min walk testing is more sensitive than maximal incremental cycle testing for detecting oxygen desaturation in patients with COPD. Chest 2003; 123: 1401-7.
32. Díaz O, Cuélar MC, Jara NM, Aravena C, Andresen M, LisBoA C. Cambios inducidos por oxígeno en la capacidad inspiratoria en reposo predicen mejoría de la capacidad de ejercicio en pacientes con EPOC. Arch Bronconeumol 2004; 40 (Supl 4): 32.

33. SNIDER GL. Enhancements of exercise performance in COPD patients by hyperoxia. A call for research. Chest 2002; 122: 1830-6.

34. Díaz O, Begin P, Torrealba B, Jover E, Lisboa C. Effects of noninvasive ventilation on lung hyperinflation in stable hypercapnic COPD. Eur Respir J 2002; 20: 1490-8.

35. Wijkstra PJ, Lacasse Y, Guyatt GH, Casanova C, Gay PC, MeEcham Jones J, Goldstein RS. A meta-analysis of nocturnal noninvasive positive pressure ventilation in patients with stable COPD. Chest 2003; 124: 33743.

36. Díaz O, Begin P, Andresen M, Prieto ME, Castilo C, Jorquera J, LisBoa C. Physiological and clinical effects of diurnal noninvasive ventilation in hypercapnic COPD. Eur Respir J 2005; 26: 1016-23.

37. Kadikar A, Maurer J, Kesten S. The six minute walk test: a guide to assessment for lung transplantation. J Heart Lung Transplant 1997; 16: 313-9.

38. Szekely LA, Oelberg DA, Wright C, Johnson DC, Wain J, Trotman-Dickerson B ET AL. Preoperative predictors of operative morbidity and mortality in COPD patients undergoing bilateral lung volume reduction surgery. Chest 1997; 111: 550-8.

39. Martínez Fj, Foster G, Curtis JL, Criner G, Weinmann G, Fishman A ET AL. Predictors of mortality in patients with emphysema and severe airflow obstruction. Am J Respir Crit Care Med 2006; 173: 1326-34.

40. Pérez G, Pinto C, Díaz O, Lisboa C. Deterioro clínico y funcional en pacientes con enfermedad pulmonar obstructiva crónica que han dejado de fumar. Rev Chil Enf Respir 2006; 22: 267.

41. Celu BR, Cote CG, Marín JM, Casanova C, Montes De Oca M, Méndez R, Pinto Plata V, Cabral HJ. The body -mass index, airflow obstruction, dyspnea and exercise capacity index in chronic obstructive pulmonary disease. N Engl J Med 2004; 350: 1005-12.

42. Cоте CG, Сеш BR. Pulmonary rehabilitation and the BODE index in COPD. Eur Respir J 2005; 25: 630-8.

43. InFeld S, Bloch KE, Weder W, Russi EW. The Bode Index after lung volume reduction surgery correlates with survival. Chest 2006; 129: 873-8. 